\title{
Aortic Root Dilatation and Marfan's Syndrome: An Alternative Diagosis
}

\author{
Sheraz Younas*, MBChB, Michael Coupe ${ }^{\dagger}$, MD, Shamyla Younas**
}

\section{INTRODUCTION}

In 1896, paediatrician, Dr Antoine Marfan, described a 5-year-old child with arachnodactyly to the Medical Society of the Paris Hospitals. Other features of Marfan's Syndrome were described over the subsequent years, including ectopia lentis in 1914, autosomal dominant inheritance, 1931, and thoracic aortic root dilatation in 1943.

Before composite graft-valve replacement was introduced as a viable prophylactic measure by Bentall and De Bono in 1968, many patients died from aortic aneurysm rupture.

Current diagnosis of a thoracic aortic aneurysm is based on echocardiographical evidence of root dilatation and aortic regurgitation at the sinuses of Valsalva with or without left heart abnormality. However, a clinical diagnosis of Marfan's Syndrome is more difficult due to ambiguity of the current clinical diagnosis criteria, its relatively sparse admissions in hospitals, and the interfamilial and intra-familial variance that the syndrome exhibits.

Accordingly, an inconspicuous Marfan's Syndrome patient, having an increased risk of aortic root aneurysm, dissection or rupture, may not be appreciated early enough to perform prophylactic valve replacement.

Increased awareness of Marfanoid signs amongst clinicians is imperative and should not be overlooked over commoner causes of aortic root regurgitation. Such surveillance will reduce the death rate associated with Marfan's Syndrome.

We discuss the diagnosis of Marfan's Syndrome in the context of a clinical scenario, which highlights this

* To whom correspondence should be addressed: Sheraz Younas, The University Of Manchester, The Stopford Building, Oxford Road, Manchester, UK

Email: sherazyounas@yahoo.co.uk

$\dagger$ Cousultant cardiologist, Royal Oldham Hospital Manchester, UK

** Medical student, University of Manchester, UK syndrome as an important cardiovascular differential diagnosis.

\section{CASE REPORT}

A 43-year-old heavy-smoking gentleman was admitted to hospital with shortness-of-breath at rest, difficulty sleeping and a two-week history of a productive lower respiratory tract infection (LRTI). He did not show any overt Marfanoid signs albeit a height of six foot and BMI $17.96 \mathrm{~kg} / \mathrm{m} 2$ (NR $20-25 \mathrm{~kg} / \mathrm{m} 2$ ).

He had never suffered from any significant illnesses and was not taking any regular medication. He lead an active life, playing squash and football.

He visited his doctor who prescribed a course of antibiotics. He failed to complete the course because he had not slept well for three weeks and felt very weak.

He later visited A\&E and a diagnosis of LRTI and panic attacks was made. He was treated accordingly.

On discharge, the patient's condition deteriorated and he was admitted again to hospital with acute haemoptosis. On investigation, a decresendo diastolic murmur was found. A routine chest x-ray was reported to be normal. An echocardiogram followed and a gross aortic root dilatation was discovered. He had a trileaflet, non-stenotic aortic valve, torrential aortic valve regurgitation, mild mitral valve regurgitation with moderate dilatation and global hypokinesis of the left ventricle. His aortic root dilated to a massive $77.2 \mathrm{~mm}$ (upper limit $=1.9 \mathrm{~cm} / \mathrm{m} 2$ of body surface area). A left heart catheterisation confirmed these findings.

His LRTI resolved and he underwent a successful composite graft-valve replacement.

During his stay, it was understood that his 11 year-old daughter exhibited Marfanoid characteristics (her height, 5 foot 8 inches and suffered from a mild functional aortic regurgitation). A thorough family history revealed his 40 year-old wife and his sisters (50 and 43 years old) were no taller than 5 foot 4 inches. His 
parents did not suffer from Marfan's Syndrome but his maternal grandfather did, who was 6 foot tall and died from cardiovascular consequences of aortic valve regurgitation.

He was slightly reticent in giving up this information because he did not think that it mattered.

\section{DISCUSSION}

\section{Marfan's Syndrome: A Differential Diagnosis}

Differentials in a case of shortness-of-breath with clinical evidence of aortic regurgitation, with or without aortic root aneurysm, include:

1. Congenital Abnormality

2. Valvular Disease:
a. Rheumatic Fever
b. Infective Endocarditis
c. Rheumatic Arthritis
d. SLE
e. Pseudoxanthoma Elasticum
f. Appetite Suppressants

3. Aortic Root Disease
a. Hypertension
b. Trauma
c. Aortic Dissection
d. Seronegative Arthritides
e. Marfan's Syndrome
f. Osteogenesis Imperfecta
g. Syphilitic Aortitis

Marfan's Syndrome is a significant differential diagnosis in this scenario, yet other connective disorders can either mask or exhibit comparable MFS characteristics; for example, Ehlers-Danlos syndrome and Lujan-Fryns Syndrome. Autosomal dominant Shprintzen-Goldberg Syndrome often exhibits Marfanoid features and may even be a variant of MFS. Homocystinuria and Beals Syndrome (FBN2 mutation) show Marfanoid signs but with ear pathologies. MASS Syndrome, a forme fruste of MFS, presents with myopia, mitral valve prolapse, aortic dilatation, skin and skeletal involvement and may be associated with FBN1 mutations. Notably, aortic dilatation is mild and the risk of aneurismal rupture is low (4). Investigations into cases of autosomal dominant ectopia lentis have shown that some patients exhibit Marfanoid features or FBN1 mutations but lack cardiac involvement.

At this point, the overwhelming array of conditions may prove diagnosing MFS difficult due to overlapping features. Similarly, in this scenario, the chest radiograph was reported to be normal, opening up the possibility of misdiagnosis. Correct clinical diagnosis is thus important in the first instance, given that results of molecular studies will only then be sought and prophylactic treatment organised as necessary.

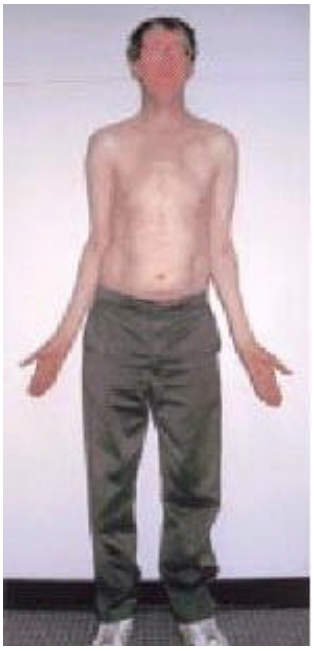

Figure 1. Patient with Marfan's Syndrome. Note pronounced marfanoid body habitus. Residual evidence of pectus carinatum can be seen. Adapted from John CS Dean. Management of Marfan's Syndrome. Heart 2002;88:97-103

\section{Marfan's Syndrome}

Marfan's Syndrome (MFS) is an inherited autosomal dominant connective tissue disorder, transmitted with full penetrance. It has a prevalence of $1 / 50003$, with pleiotropic manifestations and marked inter-familial and intra-familial variability. It lacks a predilection for race or geographic location. Seventy five percent are diagnosed through molecular studies, locating a mutation at chromosome 15q21.1 whilst linkage studies are unremarkable in 25\% of Marfanoid patients, suggesting sporadic mutation.

Two homologous genes encode the fibrillin proteins: FBN1 on chromosome 15 (15q15-32) and FBN2, chromosome 5 (5q23-31) (1). These genes encode large, cysteine-rich glycoproteins, mainly organized into calcium-binding epidermal growth factor (cbEGF)-like, EGF-like and cysteine-rich domains. Fibrillin, one of the main constituents of microfibrils, primarily represents the basal network for tropoelastin growth in elastic tissue and it also acts as an anchoring structure in nonelastic tissues (2).

The majority of FBN1 mutations detected so far are missense mutations and are probably as a result of slippage mispairing at the replication fork, primarily affecting EGF-like domains. Studies have also discovered a locus for an MFS-like disorder (3p24.2p25) in patients with isolated features of MFS but who do not fulfill the diagnostic criteria of MFS.

Mutations in FBN2 primarily cause congenital contractural arachnodactyly. Affected patients are distinguished by specific crumpling of the helix of the ear and lack of ocular complications. Production of abnormal fibrillin-1 monomers prevent normal fibrillin being encoded on the normal fibrillin gene (1) by 


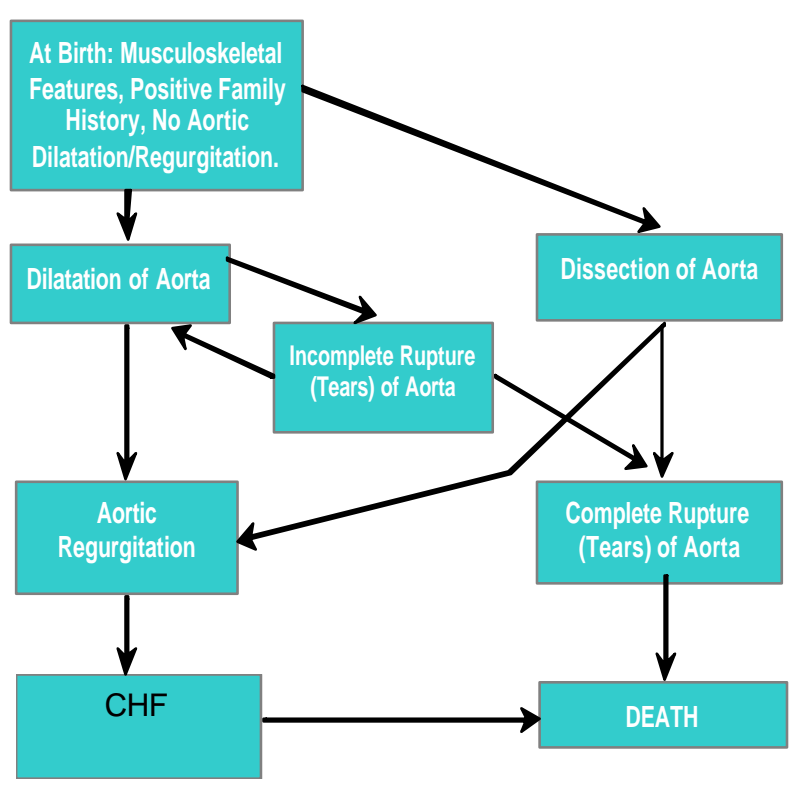

Figure 2 Scheme of development of cardiovascular complications in Marfan's Syndrome. Adapted from Fuster et al. The Heart. 10th Ed. Vol. 2. pp2160

disrupting its polymerisation: the dominant-negative effect.

The recognized systems affected in MFS are ocular, neurological, skeletal, cardiovascular, respiratory as well as the integument and dura (Fig.1). Some manifestations are age dependent.

Special attention must be directed to changes in the aortic root, the main cause of mortality. Abnormal fibrillin reduces the compliance and distensibility of the aortic wall to luminal ejection forces and associated increased pulse-wave velocity (seen by echocardiography or gated-MRI scanning). Consequently, progressive aortic dilatation, functional aortic regurgitation and eventual aortic dissection and rupture ensue; their risk increasing when the aortic root width exceeds $55 \mathrm{~mm}$ (3). On histological analysis, 'cystic medial degeneration' is typically seen as deposition of collagen and mucopolysaccharides between medial cells with elastic fibre fragmentation and paucity of smooth muscle cells. Secondary consequences include mitral valve dysfunction, left ventricular dilatation, pulmonary artery dilatation and cardiac failure. Myocardial infarction is possible if coronary ostia become occluded (Fig.2).

Deficient fibrillin deposition also leads to reduced structural integrity of the lens zonules, ligaments, pulmonary airways and spinal dura.

\section{Diagnosis}

The 1986 Berlin Nosology of Heritable Disorders of Connective Tissue (4) (appendix 1) was the main mode of diagnosis of MFS. It stipulated the involvement of

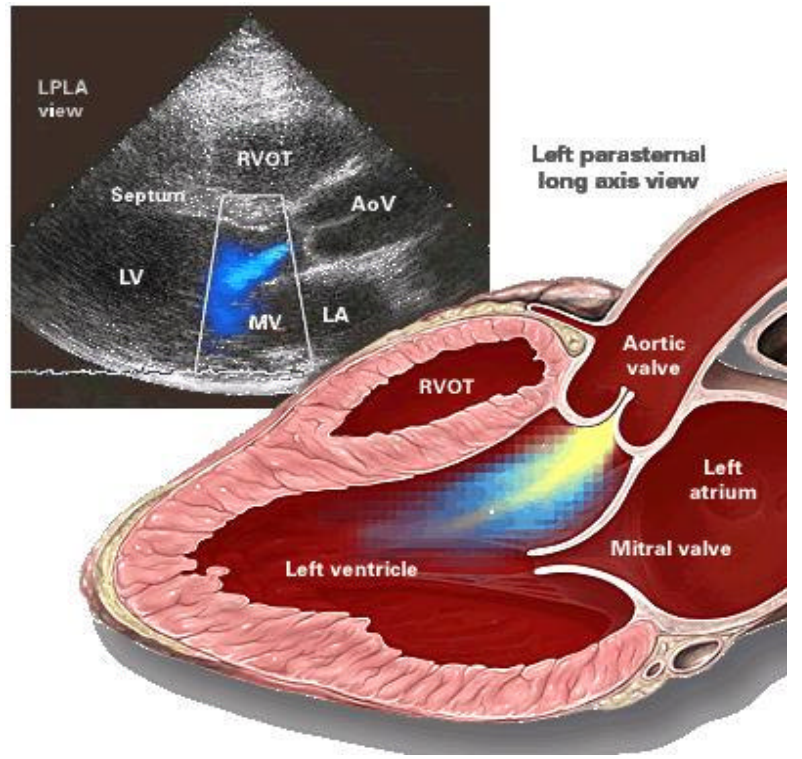

Figure 3 A typical Doppler echocardiograph in a MFS patient. The aortic valve regurgitation is apparent. The graphic shows its nature. Adapted.

the skeletal system and two other systems, with the requirement of at least one major manifestation (ectopia lentis, aortic dilatation or dissection, or dural ectasia).

In 1996, the Berlin nosology was revised to appreciate molecular data and family history. It became known as the Ghent nosology (appendix 2). Its intention was to serve as an international standard for clinical and molecular studies and for investigations of genetic heterogeneity and genotype-phenotype correlations (3). It identified the major and minor diagnostic findings.

However, the new criteria are, in some instances, found to be too stringent despite positive findings on computer tomography and magnetic resonance imaging. This highlights the ambiguity and the subjective nature in a clinical diagnosis of MFS.

Diagnostic gene sequencing (a tedious and expensive process) can identify an FBN1 mutation. Linkage analysis can track a mutated FBN1 gene within a family but a finding may represent normal variation, resulting in both false-positive and false-negative results.

At this point, no single gene probe or group of probes have been developed to detect most FBN1 mutations. Consequently, no molecular diagnosis is currently available commercially (3).

Non-invasive imaging supplements diagnosis and in some centres remains the method of quantification of the severity of cardiovascular disease, thereby aiding timely prophylactic surgical intervention.

Plain radiographs may depict medistanium widening or protrusio acetabula. Cross-sectional, transoesophageal and Doppler echocardiography will delineate heart chambers and grade valve function. It 

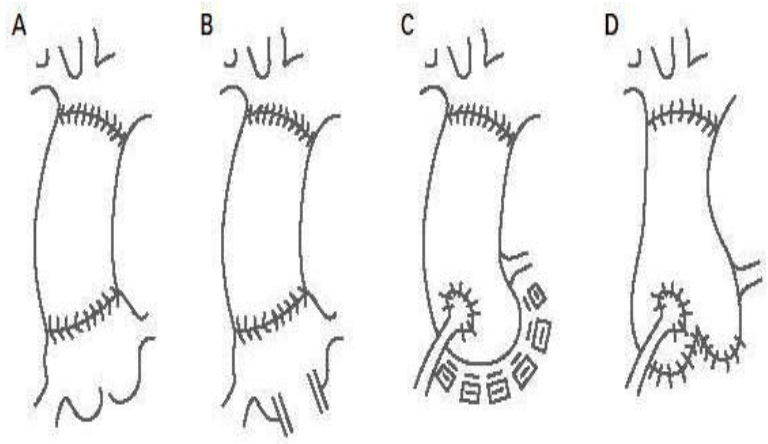

Figure 4. Operations for aortic dissection or ascending aortic replacement. (A) Simple tube replacement of the aorta for the sinotubular junction to the brachiocephalic origin. (B) Tube graft replacement and aortic valve replacement as separate components of the operation. (C) Composite graft replacement. (D) Leaflet-sparing aortic root replacement. Adapted from Tom Treasure. Cardiovascular Surgery for Marfan's Syndrome. Heart 2000;84:674-678

also aids visualisation of the aorta and measurement of the width of the sinuses of Valsalva. In MFS, the valve commissures are attached to an aorta of much greater circumference than at the nadir of the leaflet attachment; the leaflets no longer co-apt and aortic regurgitation ensues (Fig. 3) (5).

MRI favours imaging of aortic dissections. It should be performed on any patient who has actual-to-predicted aortic root dimension ratios greater than 1.5.

Considered to be the mainstay of diagnosis of aortic dissection, the sensitivity of aortography is not $100 \%$ and has its associated risks.

Immunohistologic evaluation of the skin for abnormal fibrillin is unpopular because of the high incidence of false-positive results in patients with other connective tissue disorders expressing Marfanoid characteristics (3).

\section{Management}

Management of MFS is essentially a multidisciplinary one (specific management is detailed in appendix 3 ).

\section{Beta-Blockade, ACE inhibitors, Angiotensin II blockers}

The risk of aortic dissection is intimately linked to mortality in MFS patients, and in the early 1970s, it was suggested that the reduction of the systolic impulse using -blockers may reduce this risk. Initial studies on turkeys and subsequent studies on MFS patients showed a reduced risk of dissection and fewer aortic complications with propanolol therapy, but noted a varied response to the treatment (6). Further studies have revealed that beta-blockade increases aortic distensibility and reduces aortic stiffness and pulse wave velocity $(7,8,9)$. It also showed that the group who responded to -blockade tended to have smaller aortic diameters, suggesting increased efficacy in younger

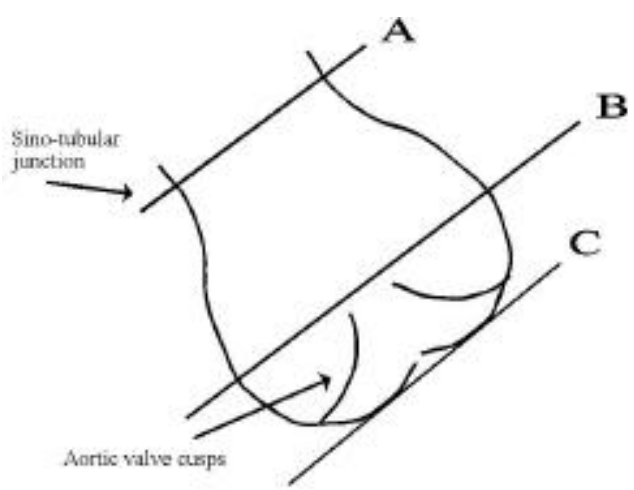

Figure 5. Echocardiographical measurements are made at A, B and $\mathrm{C}$ but the width at $\mathrm{B}$ determines the need for surgical intervention.

patients with smaller aortas $(<40 \mathrm{~mm}$ in one study (10)). Such studies provide strong evidence that -blockade should be considered in all Marfan patients, particularly in the younger age-group (4).

However, beta-blockade is not for everyone. Obvious contraindications are in patients with asthma, cardiac failure or bradyarrythmias. Instead, calcium antagonists, ACE-inhibitors or angiotensin II blockers may prove beneficial, particularly when the latter have been implicated in inhibiting vascular smooth muscle cell apoptosis, a process involved in cystic medial degeneration (11).

\section{Emergency Surgery}

Dissection of the aortic aneurysm is an absolute indication for surgical replacement. The standard operation involves replacement of the sinuses of Valsalva and as much of the ascending aorta as possible.

\section{Elective Root Replacement}

This option requires careful consideration of necessity, surgical risk, post-operative mortality rate and extent of aortic regurgitation.

The aortic root is replaced by the modified Bentall (composite graft-valve replacement) procedure (Fig. 4). This involves replacement of the sinuses, the aorta up to the innominate artery and button anastomoses of the coronary arteries. The excess (pathological) aorta, once used to contain the bleeding around suture lines, is resected and haemostasis gained under direct supervision.

An increasingly popular conservative operation, though not without criticism, involves resection of the aorta to the nadir of the aortic leaflets and attachment of the tube-graft proximally, so the valve operates within it. This method conserves the aortic valve and reverses the functional aortic regurgitation, on the premise that the aortic leaflets are without pathology.

A pertinent question is one of the timing of the replacement. Currently, there is no confident method to 
predict the timing of a dissection. Elective aortic root replacement is a pre-emptive measure and its necessity is assessed by aortic root widths6. Measurement at the level of the tips of the valve leaflets is the most accurate (Fig. 5). As a rule, a width of $60 \mathrm{~mm}$ will present a $10 \%$ risk of rupture within the next year, irrespective of site or aetiology 6. Current guidelines advocate prophylactic replacement when the width is $55 \mathrm{~mm}$ in adults; $50 \mathrm{~mm}$ in children. It is important to be weary that though the surgical risk may be lower than the risk of aortic dissection or rupture, post-operative mortality secondary to infection, false aneurysm formation, coronary anastomotic problems, anticoagulant bleeding and valve thrombosis is significant. Similarly, the rate of aortic root dilatation is important. A significant increase in diameter per six-month echocardiography (e.g. $3 \mathrm{~mm}$ ) should be promptly treated without waiting for the diameter to reach $55 \mathrm{~mm}$ (12).

The surgical indications for aortic regurgitation do not alter in an MFS patient. Valve replacement is indicated if there are symptoms attributable to regurgitation and/or evidence of an increase in left ventricular end systolic dimensions with possible adverse effects to the left ventricle or patient's prognosis. It is also advisable to replace a 'Marfan' aorta at the same time as the valve replacement, particularly if there is no reason to avoid it (6).

\section{Possible Advances}

Possible advances in treatment of vascular disease in MFS include drugs that inhibit matrix-degrading enzymes formed in inflammation. Similarly, drugs that inhibit TGF have proved successful in reversing the abnormal lung structure in mice.

Currently, studies into using an FDA-approved drug, Losartan, may also adequately suppress TGF- signalling and thus, reverse lung problems, skeletal overgrowth, valve malformation and aortic aneurysm. It has also been found that drugs stimulating fibrillin production may benefit MFS patients, despite the mutated FBN protein too being formed (13).

\section{CONCLUSION}

MFS is a differential diagnosis for shortness-of-breath and more specifically, aortic regurgitation. It carries a high risk of mortality if left undiagnosed. The benefits of acquiring a thorough history and investigations are threefold. A full family history may well reveal a genetic condition affecting the patient, e.g. MFS. Early diagnosis of aortic dilatation will alert doctors not to initiate thrombolysis in a MFS patient who had an MI, thus preventing aortic rupture. Thirdly, medicolegal issues regarding failure to recognise signs of aortic dissection, failure to inform affected patients to limit strenuous exercise and failure to advise patients and their family of reproductive risks can be avoided. Timely diagnosis of MFS can save lives.

\section{REFERENCES}

1. Dietz HC, Pyeritz RE. Mutations in the human gene for fibrillin1 (FBN1) in the Marfan syndrome and related disorders. Human Molecular Genetics 4: 1799-11809; 1995

2. Sumera N. Hasham et al. Genetic Basis Of Thoracic Aortic Aneurysms And Dissections. Opin Cardiol 2002, 17:677-683

3. John CS Dean. Management of Marfan Syndrome. Heart 88:97-103; 2002

4. Beighton P, De Paepe A, Danks D, et al. International Nosology of Heritable Disorders of Connective Tissue. Am J Med Genet 29:581-94; 1988

5. Tom Treasure. Cardiovascular Surgery for Marfan Syndrome. Heart 84:674-678; 2000

6. Shores J, Berger KR, Murphy EA, et al. Progression of aortic dilatation and the benefit of long-term -adrenergic blockade in Marfan's Syndrome. N Engl J Med 330:1335-41; 1994

7. Groenink M, Lohuis TAJ, Tijssen, et al. Survival And Complication Free Survival In Marfan's Syndrome: Implications Of Current Guidelines. Heart 82: 499-450; 1999

8. Haouzi A, Berglund H, Pelikan PCD, et al. Heterogenous aortic response to acute beta-adrenergic blockade in Marfan's Syndrome. Am Heart J 133:60-3; 1997

9. Groenink M, de Roos A, Mulder BJM, et al. Changes In Aortic Distensibility And Pulse Wave Velocity Assessed With Magnetic Resonance Imaging Following Beta-Blockade Therapy In The Marfan Syndrome. Am J Cardiol 82:203-8; 1998

10. Rios AS, Silber EN, Bavishi N, et al. Effects of long-term betaadrenergic blockade on aortic root complicance in patients with Marfan Syndrome. Am Heart J 137:1057-61; 1999

11. Nagashima $\mathrm{H}$ et al. Angiotensin II Type 2 Receptor Mediates Muscle Cell Apoptosis In Cystic Medial Degeneration Associated With Marfan's Syndrome. Circulation 104(suppl I):I-282-7; 2001

12. Treasure $\mathrm{T}$ et al. The Timing Of Aortic Root Replacement In The Marfan Syndrome: Computerised Decision Support. In: Enker J, Ed. Cardiac Surgery And Concomitant Disease. Berlin: Springer; 1999.

13. Hal Dietz, M.D. Current Research. National Marfan Foundation Journal. Vol 17

Sheraz Younas read Medicine at the University of Manchester, UK and was of a small group who were awarded scholarships. He has commenced his Pre-registration House Officer post in Wythenshawe Hospital, a UK Regional Cardiology and Cardiothoracics Centre. His area of research is Interventional Cardiology and has successfully published a paper on the efficacy of Drug Eluting Stents (Multi-centre Trials) with his colleagues. 
Appendix 1. Berlin Nosology

An diagnosis of Marfan's Syndrome requires :

If there is No family History:

Involvement of Skeleton and 2 other systems

At least 1 major manifestation

If there is an unequivocally affected $1^{\circ}$ relative:

Involvement of at least 2 systems

Requirement for a major manifestation depends on family phenotype
Table of Definitions

\begin{tabular}{ll}
\hline Systems & Major Manifestations \\
\hline Skeletal & None \\
Ocular & Ectopia Lentis \\
Cardovascular & Dilatation Of Ascending Aorta \\
Aortic Dissection & None \\
Pulmonary & None \\
Skin And Integument & None \\
Central Nervous System & Dural Ectasia \\
\hline
\end{tabular}

Appendix 2. Ghent Nosology

An diagnosis of Marfan's Syndrome requires :

Either: $\quad$ A major criterion in at least 2 systems AND Involvement of a third system

Or:

Mutation known to cause Marfan's syndrome in others AND

One major criterion in 1 system AND

Involvement of a second system

Table of Definitions

\begin{tabular}{lll}
\hline Systems & Features required to be considered a Major Criterion & System Involved \\
\hline Skeletal & $>=4 / 8$ major features & 2 major OR \\
& & 1 major and 2 minor features \\
Ocular & Ectopia lentis, myopia, global axial lengthening & 2 minor features \\
Cardiovascular & $\begin{array}{l}\text { Dilatation of ascending aorta involving Sinus of Valsalva } \\
\text { Dissection of ascending aorta }\end{array}$ & 1 minor feature \\
Pulmonary & None & 1 minor \\
Skin/integument & None & 1 minor \\
& & No additional features \\
Dura & Lumbosacral dural ectasia & No additional features \\
Family/genetic history & -1 st degree relative who can be diagnosed affected without family history \\
& - Presence in the patient of Fibrillin mutation known to cause Marfan's syndrome
\end{tabular}


Appendix 3. The Marfan's Syndrome Clinical Guideline

INITIAL ASSESSMENT AND DIAGNOSIS

" The diagnosis of Marfan's syndrome should be made on the basis of the Ghent criteria.

" The initial assessment should include the following: personal history, detailed family history, clinical examination including ophthalmology examination, transthoracic echocardiogram.

" The echocardiogram should include measurement of the aortic diameter at the Sinus of Valsalva, and this dimension should be related to normal values based on age and body surface area.

" The development of scoliosis and protrusio acetabulae and dural ectasia is age dependent. X-ray or MRI examination for these features should be considered in those of the appropriate age, if a positive finding would make the diagnosis of Marfan's or if other clinically important decisions depend on the findings.

" Younger patients with a positive family history who do not manifest sufficient clinical features to fulfill the diagnostic criteria for affected status, and in whom DNA testing is not successful, should have further clinical evaluations pre-school, before puberty and at age 18 or more frequently, particularly around puberty, as the clinical situation dictates.

" Younger patients with no family history, who fall short of fulfilling the diagnostic criteria by one system only should have further evaluations pre-school, at puberty and at age 18 or more frequently, particularly around puberty, as the clinical situation dictates.

\section{MANAGEMENT: CARDIOVASCULAR SYSTEM}

" Beta blocker therapy should be considered in any Marfan patient with aortic dilatation at any age.

" Marfan patients should be referred for consideration of prophylactic aortic root replacement when the aortic root at the Sinus of Valsalva exceeds $5 \mathrm{~cm}$ in an adult or child.

" Marfan patients of all ages should be offered at least annual evaluation with clinical history, examination and transthoracic echocardiography. In children, serial trans thoracic echocardiography at 6-12 month intervals is recommended, the frequency depending on the actual aortic diameter (in relation to body surface area) and the rate of increase.

\section{MANAGEMENT: SKELETAL SYSTEM}

" Even if there is no clinical scoliosis children should have an erect A-P plain X-ray film of the spine i.e. between the ages of 9 - 11 years of age. " A child with a clinical scoliosis requires formal orthopaedic assessment, and 6 monthly orthopaedic follow-up until the growth spurt is completed is recommended (Pyeritz 1979).

" Adults with angle of curvature is $>40^{\circ}$ should be referred to the orthopaedic department for indefinite follow-up (minimum yearly).

" Contact sports and diving should be avoided to reduce the risk of aortic rupture.

\section{MANAGEMENT: OCULAR SYSTEM}

" When there is a family history, initial examination by an ophthalmologist should take place at three to six months of age to look for evidence of lens dislocation, anterior chamber problems and incipient retinal detachment.

" When there is no family history, examination by an ophthalmologist should form part of the initial clinical assessment of the patient.

" To detect later changes in refraction in Marfan's patients, such as those caused by later childhood lens subluxation, and to prevent amblyopia, annual review by an orthoptist or optometrist until the age of 12 is recommended.

" Families should be informed about the risk of retinal detachment and glaucoma, to that they can seek appropriate advice promptly should symptoms develop.

\section{MANAGEMENT: RESPIRATORY SYSTEM}

" Pulmonary function tests should form part of the assessment of pectus deformity.

" Surgical repair of pectus excavatum should be delayed, if possible, until late adolescence, and should include internal stabilisation to reduce the risk of recurrence.

" If cardiac and pectus surgery are required as elective procedures, surgical repair of pectus excavatum should precede cardiac surgery by several months for optimal functional and cosmetic results.

Adapted from John C S Dean Et Al. Clinical Guidelines and an Integrated Care Pathway In Marfan Syndrome. Dr John C S Dean, Department Of Medical Genetics, Medical School, Foresterhill, Aberdeen AB25 9ZD, Scotland, UK 